\title{
Multilayer extreme learning machine for hand movement prediction based on electroencephalography
}

\author{
Khairul Anam ${ }^{1}$ Cries Avian², Muhammad Nuh ${ }^{3}$ \\ ${ }^{1,2}$ Department of Electrical Engineering, Universitas Jember, Indonesia \\ ${ }^{3}$ Department of Biomedical Engineering, Institut Teknologi Sepuluh November, Indonesia
}

\begin{tabular}{l} 
Article Info \\
\hline Article history: \\
Received Dec 10, 2019 \\
Revised Mar 29, 2020 \\
Accepted May 3, 2020 \\
\hline Keywords: \\
Classification methods \\
Electroencephalography \\
Extreme learning machine \\
Movement prediction \\
Multilayer ELM
\end{tabular}

Article Info

Article history

Received Dec 10, 2019

Revised Mar 29, 2020

Accepted May 3, 2020

Multilayer ELM

\begin{abstract}
Brain computer interface (BCI) technology connects humans with machines via electroencephalography (EEG). The mechanism of $\mathrm{BCI}$ is pattern recognition, which proceeds by feature extraction and classification. Various feature extraction and classification methods can differentiate human motor movements, especially those of the hand. Combinations of these methods can greatly improve the accuracy of the results. This article explores the performances of nine feature-extraction types computed by a multilayer extreme learning machine (ML-ELM). The proposed method was tested on different numbers of EEG channels and different ML-ELM structures. Moreover, the performance of ML-ELM was compared with those of ELM, Support Vector Machine and Naive Bayes in classifying real and imaginary hand movements in offline mode. The ML-ELM with discrete wavelet transform (DWT) as feature extraction outperformed the other classification methods with highest accuracy 0.98. So, the authors also found that the structures influenced the accuracy of ML-ELM for different task, feature extraction used and channel used.
\end{abstract}

This is an open access article under the CC BY-SA license.

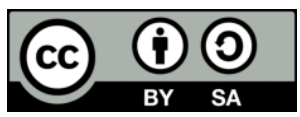

\section{Corresponding Author:}

Khairul Anam,

Department of Electrical Engineering,

Universitas Jember,

Jl. Kalimantan No.37, Sumbersari, Jember, East Java, Indonesia.

Email: khairul@unej.ac.id

\section{INTRODUCTION}

Technological advances, especially in Brain-Computer Interfaces (BCIs), play a crucial role in assisting patients with physical disabilities or paralysis [1]. BCI detects the brain activity in the cortex as electroencephalography (EEG) signals. One of the most popular BCI applications is classifying human motor movements, especially hand movements, for various assistive robots [2, 3]. Hand movements can be recognized and classified by various recognition/classification methods $[4,5]$ that distinguish between rightand left-hand movements in real-time [6, 7] or in imagery [8, 9]. The pattern recognition method must be optimized before implementing the EEG signal on an assistive robot that controls the robot hand/robot therapy.

In EEG signal processing, the signal information is first extracted and then classified [9]. Among the most popular classification methods are the support vector machine (SVM) [10, 11], linear discriminant analysis [12, 13], artificial neural networks [14, 15], and fuzzy algorithms [16, 17]. The accuracy obtained in each classification method also varies in each study, like the results obtained by Farooq et al. [18]. In this research, the classification method used the principal component analysis (PCA) and independent component analysis (ICA) as feature extraction with the $\mathrm{K}$ nearest neighbor $(\mathrm{KNN})$ as a classifier which obtained an accuracy 
rate of $80.6 \%$. Besides that, Kevric et al. [19] also use KNN as its classifier with discrete wavelet transform (DWT) as feature extraction. From this study, the accuracy of the system reaches $92.8 \%$. Deep learning was also used in this case, one of which was a study conducted by Korhan et al. [20] who tried to solve problems using common spatial filter (CSP) and convolutional neural network (CNN). In this study, the system reaches an accuracy of $93.75 \%$. However, the existing classification methods often struggle to meet the following requirements: (1) ease of use, (2) low computational complexity, and (3) high accuracy [1, 21]. One classification methods that can achieve these criteria is the extreme learning machine (ELM) [22].

The ELM was applied to EEG classification by Tan et al. [23]. They reported that ELM delivers excellent performance compared to other classification methods. However, other studies have reported poor performance stability in ELM. The poor performance is caused by the randomness of assigning weights $(w)$ to the neurons and the threshold between the input layer and the hidden layer [24]. These problems degrade the accuracy of ELM classification. However, the problems found in ELM have been resolved by the existence of a multilayer extreme learning machine (ML-ELM). With its multi-layered structure, ML-ELM overcomes the problems found in ELM. In particular, the data are transformed in each layer, accelerating the convergence and minimizing the errors [24]. Research conducted by Duan et al. [25] has proven the superiority of this ML-ELM. In that study, researchers used the PCA and linear discriminant analysis (LDA) as feature extraction with the ML-ELM as the classifier. The results obtained indicate that the accuracy rate of this system is $94.2 \%$. But unfortunately, Duan et al. [25] only explored the use of ML-ELM in the extraction feature, even though many feature extractions can be used to improve the accuracy of the classification of EEG signals such as power spectral density (PSD) and also DWT. Therefore, to this end, the authors explore various feature extraction methods employed in previous studies, and integrate them into a multilayer (ML) ELM classifier.

In this exploration study, the performance of hand classification will be improved by using several step. First, the ML-ELM was paired with nine feature extractions that are popularly used in EEG. After selecting the best feature extraction-classifier pairs, we tracked the performances of the optimized ML-ELM classifier with different numbers of channels and different structures. To demonstrate the superior performance of ML-ELM, the classifier was competed against the ELM, SVM and Naive Bayes (NB) classifiers. Each of the three classifiers performed nine types of feature extractions. The nine-feature extraction was selected because in our previous research, the features used were limited to power features [23]. Feature extraction in EEG has many variations [26] which can be combined with ML-ELM. This opportunity must be exploited because different feature extraction and classification methods can pool their specific advantages to improve the accuracy of the result. The accuracy performances of the classification methods were evaluated by the K-fold technique. The ML-ELM outperformed the other classifiers. When paired with discrete wavelet density, ML-ELM delivered its highest performance, with the highest classification accuracy of $0.98(98 \%)$.

\section{RESEARCH METHOD}

\subsection{Multilayer extreme learning machine}

As explained in the previous section, ML-ELM is ELM with more than one hidden layer [27]. The last hidden layer generally processes the ELM algorithm. To qualify as ML-ELM, some articles require the ELM to possess more than two hidden layers [24]. ML-ELM differs from ELM in fundamental ways. ML-ELM is based on the ELM auto-encoder [28], and is algorithmically similar to deep learning. The ELM auto-encoder operates on the weights $(w)$ of the hidden layers during training with unsupervised learning. However, unlike deep learning, there is no fine-tuning in ML-ELM. The activation function in ML-ELM can be linear or nonlinear. If the number of nodes $L_{k}$ in the $k$-th hidden layer equals the number of nodes $L_{k-1}$ in the $(k-1)$-th hidden layer, $g$ is a linear function; otherwise, $g$ is a nonlinear connection such as a sigmoidal function. The output matrix $H^{k}$ of hidden layer $k$ is given by:

$$
H^{k}=g\left(\left(\beta^{k}\right)^{T} H^{k-1}\right)
$$

The input layer $x$ can be considered as the 0 -th hidden layer (i.e., $k=0$ ). The output of the connection between the last hidden layer and the output node $t$ is analytically calculated by the regularized least-squares method.

\subsection{Dataset and system construction}

The data in this research were provided by Hohyun Cho from the School of Electrical Engineering and Computer Science, Gwangju Institute of Science and Technology, South Korea [29]. The dataset consists of 52 data with two types of hand movements: real right- and left-hand movements, and imaginary right- and left-hand movements. The dataset contains 64 channels of EEG data. In this study, the EEG signals in this 
dataset were extracted by several signal processing methods. The methods were evaluated on nine popular feature extraction types used in existing studies as shown in Table 1.

Table 1. The nine feature-extraction types employed in the present study

\begin{tabular}{|c|c|c|c|}
\hline No & Feature Extraction & Equation & Information \\
\hline 1 & Bandpower [30] & $\begin{array}{l}\text { deltaBP }=\int_{n=1}^{\text {Fres }} w(\text { idxDelta }) \\
\text { Fres }=\frac{F_{s}}{N}=\frac{F_{s}}{F_{s} t}=\frac{1}{t}\end{array}$ & $\begin{array}{l}\text { idxDelta is the power at a specific } \\
\text { frequency (bandpass). }\end{array}$ \\
\hline 2 & Hurst Coeffecient [31] & $\mathrm{Hc}=\log (R / S) / \log (T)$ & $\begin{array}{l}T \text { is the sampling period, } R / S \text { is } \\
\text { the rescaled range value. }\end{array}$ \\
\hline 3 & $\begin{array}{l}\text { Detrended Fluctuation } \\
\text { Analysis (DFA) [32] }\end{array}$ & $F(n)=\sqrt{\frac{1}{N} \sum_{k=1}^{N}\left(x(k)-X_{n}(k)\right)^{2}}$ & $\begin{array}{l}X(k) \text { is the EEG in the time series } \\
\text { data, } X_{n}(k) \text { is the local trend. }\end{array}$ \\
\hline 4 & Hjorth [31] & $\begin{array}{l}\text { Activity }=\sigma^{2}{ }_{0} \\
\text { Mobility }=\sqrt{\left(\sigma_{2} / \sigma_{1}\right)^{2}-\left(\sigma_{1} / \sigma_{0}\right)^{2}} \\
\text { Complexity }=\sigma_{1} / \sigma_{0}\end{array}$ & $\begin{array}{l}\sigma_{0} \text { is the average signal power or } \\
\text { variance. } \sigma_{1} \text { and } \sigma_{2} \text { are variants of } \\
\text { the first and second signal } \\
\text { derivatives, respectively. }\end{array}$ \\
\hline 5 & $\begin{array}{l}\text { Power Spectral Density } \\
\text { (PSD) [30] }\end{array}$ & $\begin{array}{l}\hat{p} d(f)=\frac{1}{M U}\left|\sum_{N=0}^{M-1} x d(n) w(n) e^{-j 2 \pi f}\right|^{2} \\
U=\frac{1}{M} \sum_{n=0}^{M-1}|w(n)|^{2} \\
\hat{P} W e l c h(f)=\frac{1}{L} \sum_{n=0}^{M-1} \hat{P} d(f)\end{array}$ & $\begin{array}{l}\{x d(n)\} \text { is a sequence with } d= \\
1,2,3 \ldots L \text { signal intervals, each of } \\
\text { length } M . \quad U \text { stands for } \\
\text { the normalization factor of power } \\
\text { in the window function. } w(n) \\
\text { denotes the windowed data. }\end{array}$ \\
\hline 6 & $\begin{array}{l}\text { Mean Absolute Value } \\
\text { (MAV) [33] }\end{array}$ & $M A V=\frac{1}{N} \sum_{i=1}^{N}\left|x_{i}\right|$ & $\begin{array}{l}x \text { is a signal in a large sample of } \\
\text { size } N, i \text { is the sequence index. }\end{array}$ \\
\hline 7 & Standard Deviation [33] & $S T D=\sqrt{\frac{\sum_{i=1}^{N}\left(x_{i}-\bar{x}\right)^{2}}{N-1}}$ & $\bar{x}$ is the average of $x$. \\
\hline 8 & $\begin{array}{l}\text { Petrosian Fractal } \\
\text { Dimension (PFD) [31] }\end{array}$ & $P F D=\log _{10} k /\left(\log _{10} k+\log _{10}\left(\frac{k}{k+0.4 N \delta}\right)\right)$ & $\begin{array}{l}K \text { is the number of samples in the } \\
\text { signal. } N \delta \text { denotes the number of } \\
\text { signal changes in the signal } \\
\text { derivative. }\end{array}$ \\
\hline 9 & $\begin{array}{l}\text { Discrete Wavelet } \\
\text { Transform (DWT) [34] }\end{array}$ & $\begin{array}{l}d 1[k]=y \text { high }[k]=\sum_{n} x[n] \cdot g[2 k-n] \\
a 1[k]=y \text { low }[k]=\sum_{n} x[n] \cdot h[2 k-n]\end{array}$ & $\begin{array}{l}x[n] \text { is a discrete-time signal, } \\
g[.] \text { is first passed through a half- } \\
\text { band high-pass filter and then } \\
\text { through } h[.] \text { in the low-pass filter. } \\
d 1 \text { and al are level-1 } \\
\text { approximation coefficients. } \\
y h i g h[k] \text { and ylow }[k] \text { are the } \\
\text { outputs of the high-pass and low- } \\
\text { pass filters, respectively, after } \\
\text { subsampling. }\end{array}$ \\
\hline
\end{tabular}

Prior to extraction, the EEG signal was preprocessed through a bandpass filter operated at $8-14 \mathrm{~Hz}$ with a sampling frequency of $512 \mathrm{~Hz}$. For the present study, the authors chose the data on subjects 3, 5, 9, 14, $15,35,41,43,46$, and 52 from the provided dataset. The preprocessed and extracted EEG signals were then classified by various types of extraction methods applied in the literature. The classification methods are required to discriminate between right- and left-hand movements. This study compared the results of three classification methods: ELM, ML-ELM, SVM and NB. Figure 1 is a flowchart of the work process. The proposed system is implemented in Python 3.6.5. 


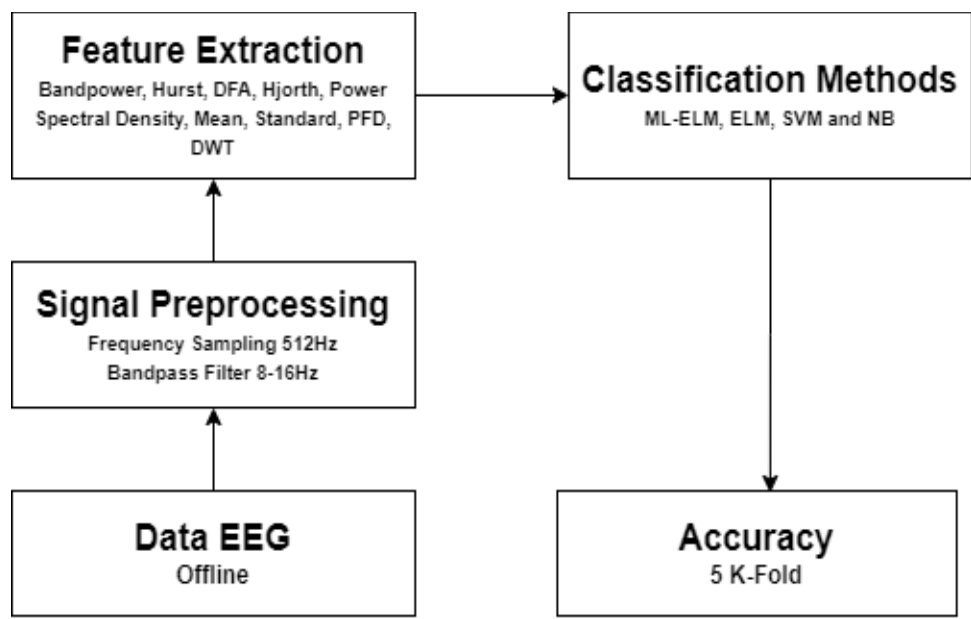

Figure 1. Methods adopted in the present research

\subsection{Testing methods and parameters}

The method was tested for different numbers of channels and different structures of the ML-ELM. The testing methods are detailed by:

Testing Method 1: The dataset was classified by pairs of classification methods. Altogether, there were 36 pairs of classification methods (nine feature extractions performed by four classifiers). Each pair was required to classify the five selected dataset subjects on $3,5,9,15,41$, or 64 channels. The details of the channels were as follows: 3 channels: $\mathrm{C} 3, \mathrm{Cz}, \mathrm{C} 4$; 5 channels: $\mathrm{C} 3, \mathrm{C} 1, \mathrm{Cz}, \mathrm{C} 2, \mathrm{C} 4$; 9 channels: $\mathrm{C} 3, \mathrm{C} 2$, CZ, C2, C4, FZ, FCz, CP2, Pz; 15 channels: FC3, FC1, FCz, FC2, FC4, C3, C1, Cz, C2, C4, CP3, CP1, CPZ, CP2, CP4; 41 channels: AF3,AFZ,AF4,F5,FC3,FC1, FCz, FC2, FC4, FC6, C5, C3, C1, Cz, C2, C4, C5, CP5, CP3, CP1, CPZ, CP2, CP4, CP6, P5, P3, P1, Pz, P2, P4, P6, PO3, POz, PO4; and 64 channels: all channels in the dataset. The number of neurons was 1000 in the ELM classifier and 1000 in each layer (five layers) of the ML-ELM classifier.

Testing Method 2: Using the two feature extractions yielding the highest accuracy and the two number of the optimal channels, the system was tested for different structures of the ML-ELM. From an initial sequence of two layers, the ML-ELM was built to a maximum of eight hidden layers with a maximum neuron number of 100 per layer. Before adding a new hidden layer, each neuron in the present layer was increased by ten neurons. All tests were performed on the ten subjects selected from the dataset. The results in the following section report the average accuracy over all subjects and all channel used, as obtained in the 5-fold evaluation.

\section{RESULTS AND DISCUSSION}

This section reports and discusses the results under the test scenarios described in the previous section.

\subsection{Testing method 1}

The results of the ML-ELM, ELM, SVM and NB methods are shown in Figures 2, 3, and 4, respectively. Figures 2 and 3 are the performance data for each pair of classification methods. The results contained in the Figures are the result of the accumulation of all subjects used and the entire channel used. The accumulated results are then averaged based on feature extraction and classifier. After that, the results are displayed in a graph contained in Figures 2 and 3. The results obtained indicate that PSD and DWT have higher performance compared to other extraction features. This claim is found in the ELM and ML-ELM classifier types. In the Movement type, PSD has the best accuracy on the combination with ML-ELM 0.92. As for DWT, the performance is owned by ELM with an accuracy of 0.95.

For imagery types, ML-ELM has the best accuracy on PSD with an accuracy of 0.93. In DWT, ML-ELM also has the best performance with a value of 0.96, while ELM has an accuracy of 0.958. Related to the performance of each classifier, then analyzing of head-to-head comparison data is needed for each task. It can be known by adding up the overall accuracy contained in the nine extraction features used. The best performance data can be seen in Figure 4. The results show that ML-ELM has the best performance compared to the other three classifiers. The total accuracy obtained by ML-ELM on the nine feature extractions are 5.47 for movement task and 5.28 for imagery task. Even so, ELM has almost the same performance as ML-ELM, with a value of 5.40 for imagery and 5.25 for movement. 


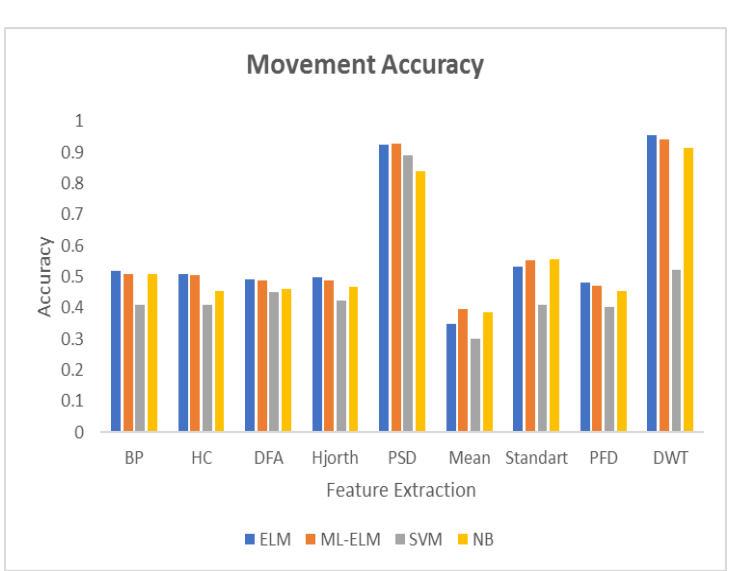

Figure 2. Performance results of each clasification method pairs in movement task

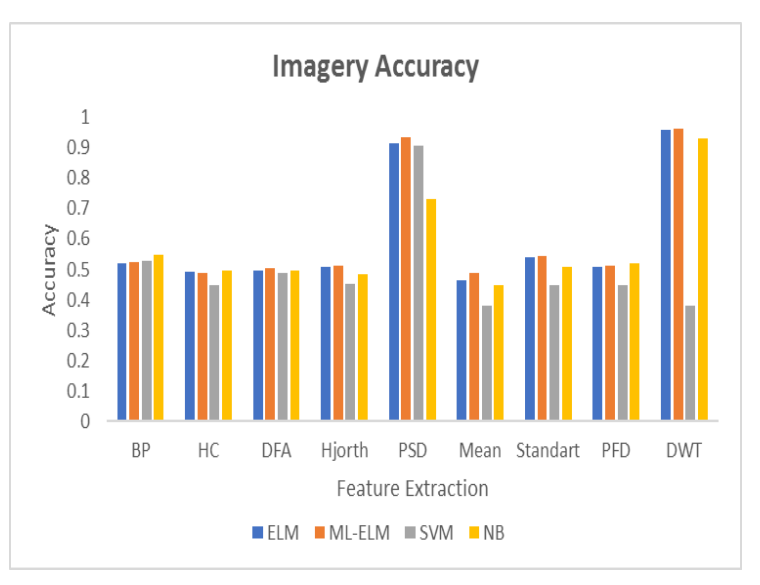

Figure 3. Performance results of each clasification method pairs in imagery task

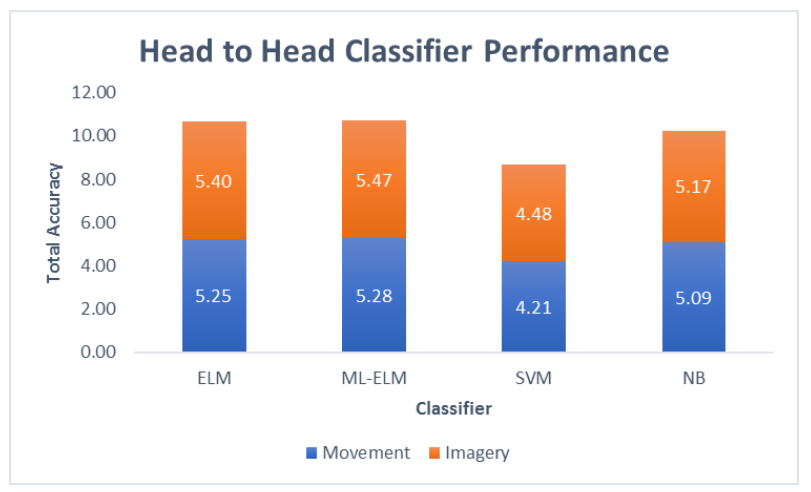

Figure 4. Total accuracy of classifier performace in each task

\subsection{Testing method 2}

This test was performed for different structures of ML-ELM. The test was intended to determine the effect of changing the number of hidden layers and neurons on the accuracy results. The results are presented in Figures 5 and 6. Labels such as "PSD-I-15" describe the feature extraction type, type of movement ("I" or "M")-number of channels (15 or 41). The numbers along the $\mathrm{x}$-axis are the combined numbers of hidden layers and neurons. The number of tens symbolizes the number of hidden layers and the unit number symbolizes the number of neurons. For example, 23 denotes three hidden layers with 1000 neurons in that layer and 30 neurons in the fourth layer. The selected numbers of channels (15 and 41) provided the highest accuracy in the previous test. The results of the present test are the average accuracies over the ten selected subjects.

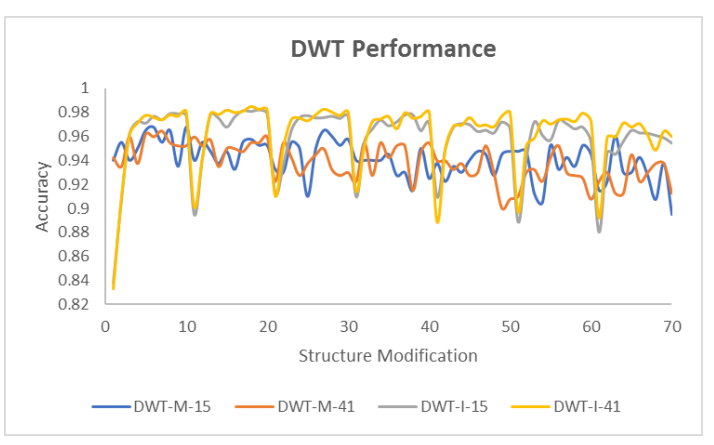

Figure 5. DWT performance in structure change of ML-ELM

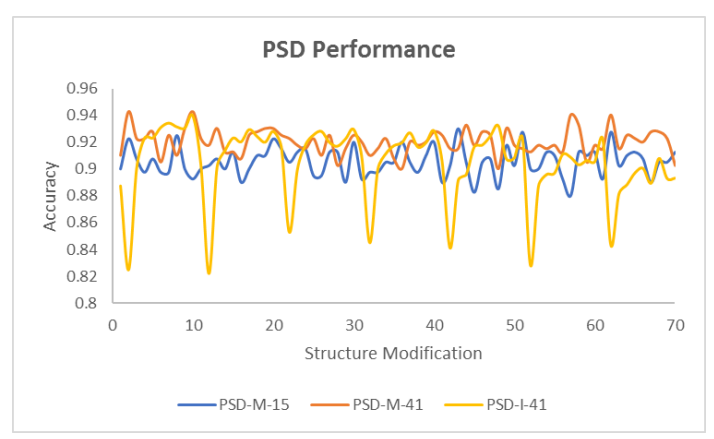

Figure 6. PSD performance in structure change of ML-ELM 
From the results obtained indicate that the ML-ELM structure has a significant effect on the level of accuracy obtained. This proves that there is a need for tuning the ML-ELM structure in different types of tasks, feature extraction and the number of channels to get the best performance. For example in the task movement data with DWT extraction feature using 41 channels, it is found that the average of the accuracy of 10 subjects, the best performance is located in the 7th ML-ELM structure or with the structure [1000, 700] which means 1000 is the first number of neurons and 700 for neurons second. Whereas in other structures, it has fluctuating accuracy. The lowest value obtained is in the 49th structure (1000 neurons for layers 1 to 5 and 900 neurons for layer 6) with an accuracy of 0.9. Whereas the type of task imagery with DWT extraction feature uses 41 channels, the best structure is in structure number 17 (1000 neurons for layers 1 to 2 and 700 neurons in layer 3) with an accuracy of 0.984 . When viewed in the use of a different number of channels, the best structure that is produced is also different. For example, on the use of channel number 15, DWT on imagery task has the best performance on structure number 6, and on the movement task has the best performance on structure number 19. This is also experienced on PSD feature extraction both on using the different number of channels and various tasks. However, when compared, DWT still has the best performance compared to PSD. When examined, the phenomenon that occurs in the form of fluctuating accuracy values is caused by underfitting and overfitting problems. This can be observed from Figure 5 and Figure 6. If with the addition of structure, the accuracy rate is getting better, then the system is underfitting. Conversely, if the addition of structure precisely decreases the accuracy level, it means the system is overfitting.

\section{CONCLUSION}

The results confirmed the superior classification performance of multilayer extreme learning machine (ML-ELM) over ELM, Support Vector Machine and Naive Bayes on almost every featureextraction evaluation. Moreover, ML-ELM delivered its best results with DWT as feature extraction. The accuracy of ML-ELM depended on the number of channels used, the feature extraction, and the task. However, the accuracy was sometimes degraded by underfitting and overfitting problems, causing accuracy fluctuations in the results of different structure change. In this article, the combinations of feature extractions and classification methods were somewhat limited. In future research, the underfitting and overfitting problem will be resolved by combining ML-ELM with optimization algorithms. The second line of research is related to combinations of feature extractions. Instead of a single feature extraction, ML-ELM can be combined with several feature extractions. This is expected to improve the accuracy by increasing the ease of differentiating the data. Finally, one could apply additional filters.

\section{ACKNOWLEDGEMENTS}

We would like to thank the Directorate Research and Development, The Ministry of Research and Higher Education, the Republic of Indonesia for research funds under Post-doctoral Research Scheme Contract no. 175/SP2H/LT/DRPM/2019.

\section{REFERENCES}

[1] L. Bi, X. Fan and Y. Liu, "EEG-Based Brain-Controlled Mobile Robots: A Survey," in IEEE Transactions on Human-Machine Systems, vol. 43, no. 2, pp. 161-176, March 2013.

[2] O. Rahma, R. Hendradi and F. Ama, "Classifying imaginary hand movement through electroencephalograph signal for neuro-rehabilitation," Walailak J Sci Technol, vol. 16, no. 12, pp. 943-953, 2019.

[3] S. A. Dewangga, H. Tjandrasa, D. Herumurti, "Robot motion control using the emotiv EPOC EEG system," Bull Electr Eng Inf, vol. 7, no. 2, pp. 279-285, 2018.

[4] R. Martín-Clemente, J. Olias, D. B. Thiyam, A. Cichocki and S. Cruces, "Information theoretic approaches for motor-imagery BCI systems: review and experimental comparison," Entropy, vol. 20, no. 1, pp. 7:1-29, 2018.

[5] N. E. Md Isa, A. Amir, M. Z. Ilyas and M. S. Razalli, "Motor imagery classification in brain computer interface (BCI) based on EEG signal by using machine learning technique," Bull Electr Eng Inf, vol. 8, no. 1, pp. 269-275, 2019.

[6] A. Dey, S. Bhattacharjee and D. Samanta, "Recognition of motor imagery left and right hand movement using EEG," 2016 IEEE International Conference on Recent Trends in Electronics, Information \& Communication Technology (RTEICT), Bangalore, pp. 426-430, 2016.

[7] K. P. Thomas, N. Robinson, K. G. Smitha and A. P. Vinod, "EEG-based Discriminative Features During Hand Movement Execution and Imagination," 2018 15th International Conference on Control, Automation, Robotics and Vision (ICARCV), Singapore, pp. 883-888, 2018.

[8] S. A. El-aal, R. A. Ramadan and N. I. Ghali, "EEG signals of motor imagery classification using adaptive neurofuzzy inference system," Advances in Nature and Biologically Inspired Computing, vol. 419, pp. 105-116, 2016. 
[9] A. Schwarz, P. Ofner, J. Pereira, A. I. Sburlea and G. R. Müller-Putz, "Decoding natural reach and grasp actions from human EEG," J Neural Eng, vol. 15, no. 1, pp. 1-23, 2017

[10] X. Tang, J. Yang and H. Wan, "A hybrid SAE and CNN classifier for motor imagery EEG classification," In Artificial Intelligence and Algorithms in Intelligent Systems, Proceedings of 7th Computer Science On-line Conference, vol 2, pp. 265-278, 2018.

[11] J. Moreira, M. Moreira, N. Pombo, B. M. C. Silva and N. M. Garcia, "Identification of real and imaginary movements in EEG using machine learning models," In CMBEBIH: International Conference on Medical and Biological Engineering, Banja Luka, pp. 469-474, 2019.

[12] N. G. Ozmen, L. Gumusel and Y. Yang, "A biologically inspired approach to frequency domain feature extraction for EEG classification," Comput Math Methods Med, vol. 2018, pp. 19-24, 2018.

[13] S. Razi, M. R. K. Mollaei and J. Ghasemi, "A novel method for classification of BCI multi-class motor imagery task based on Dempster-Shafer theory," Inf Sci, vol. 484, pp. 14-26, 2019.

[14] A. Javed, Mohsin I. Tiwana, Moazzam I. Tiwana, N. Rashid, J. Iqbal, and U. S. Khan, "Recognition of finger movements using EEG signals for control of upper limb prosthesis using logistic regression," Biomed Res, vol. 28, no. 17, pp. 7361-7369, 2017.

[15] S. Bhattacharya, K. Bhimraj, R. J. Haddad and M. Ahad, "Optimization of EEG-based imaginary motion classification using majority-voting," SoutheastCon 2017, Charlotte, NC, pp. 1-5, 2017,

[16] T. Nguyen, I. Hettiarachchi, A. Khosravi, S. M. Salaken, A. Bhatti and S. Nahavandi, "Multiclass EEG data classification using fuzzy systems," 2017 IEEE International Conference on Fuzzy Systems (FUZZ-IEEE), Naples, pp. 1-6, 2017.

[17] R. Chatterjee, T. Bandyopadhyay, D. K. Sanyal and D. Guha, "Comparative analysis of feature extraction techniques in motor imagery EEG signal classification," In: Somani A., Srivastava S., Mundra A., Rawat S. (eds) Proceedings of First International Conference on Smart System, Innovations and Computing. Smart Innovation, Systems and Technologies, vol. 79, pp. 73-83, 2018.

[18] E. J. Rechy-Ramirez and H. Hu, "Bio-signal based control in assistive robots: a survey," Digit Commun Netw, vol. 1, no. 2, pp. 85-101, 2015.

[19] C. Chen, K. Li, M. Duan and K. Li, "Extreme learning machine and its applications in big data processing," in Big Data Analytics for Sensor-Network Collected Intelligence: Intelligent Data-Centric Systems, Elsevier, 2017.

[20] P. Tan, W. Sa and L. Yu, "Applying Extreme Learning Machine to classification of EEG BCI," 2016 IEEE International Conference on Cyber Technology in Automation, Control, and Intelligent Systems (CYBER), Chengdu, pp. 228-232, 2016.

[21] D. Xiao, B. Li and Y. Mao, "A multiple hidden layers extreme learning machine method and its application," Math Probl Eng, pp. 1-10, 2017.

[22] A. R. Mane, S. D. Biradar and R. K. Shastri, "Review paper on Feature Extraction Methods for EEG Signal Analysis," Int J Emerg Trend Eng Basic Sci, vol. 2, no. 1, pp. 545-552, 2015.

[23] R. M. Parkavi, M. Shanthi and M. C. Bhuvaneshwari, "Recent trends in ELM and MLELM: a review," Adv Sci Technol Eng Syst J, vol. 2, no. 1, pp. 69-75, 2017.

[24] Kasun LLC, Zhou H, Huang G-B et al, "Representational learning with ELMs for big data. IEEE Intell Syst, vol. 4, pp. 1-4, 2013.

[25] H. Cho, M. Ahn, S. Ahn, M. Kwon and S. C. Jun, "EEG datasets for motor imagery brain computer interface," GigaScience, vol. 6, no. 7, pp. 1-7, 2017,

[26] S. Sarraf, "EEG-Based Movement Imagery Classification Using Machine Learning Techniques and Welch's Power Spectral Density Estimation," Am Sci. Res. J Eng Technol Sci, vol. 33, pp. no. 1, 124-145, 2017.

[27] M. Peker, "An efficient sleep scoring system based on EEG signal using complex-valued machine learning algorithms," Neurocomputing, vol. 207, pp. 165-177, 2016.

[28] A. Adda and H. Benoudnine, "Detrended fluctuation analysis of EEG recordings for epileptic seizure detection," 2016 International Conference on Bio-engineering for Smart Technologies (BioSMART), Dubai, pp. 1-4, 2016.

[29] A. Khorshidtalab, M. J. E. Salami and M. Hamedi Khorshidtalab A, Salami MJE, Hamedi M, "Robust classification of motor imagery EEG signals using statistical time-domain features," Physiol Meas, 34, no. 11, pp. 1563-1579, 2013.

[30] P. Ghorbanian, D. M. Devilbiss, A. J. Simon, A. Bernstein, T. Hess and H. Ashrafiuon, "Discrete wavelet transform EEG features of Alzheimer'S disease in activated states," 2012 Annual International Conference of the IEEE Engineering in Medicine and Biology Society, San Diego, CA, 2012, pp. 2937-2940. 